\title{
Professional Integrity and the Role of Medical Students in Professional Self-Regulation
}

\author{
John H. Coverdale ${ }^{1} \cdot$ Laura Weiss Roberts ${ }^{2} \cdot$ Richard Balon $^{3} \cdot$ Eugene V. Beresin $^{4}$. \\ Alan K. Louie ${ }^{2}$ - Anthony P. S. Guerrero ${ }^{5}$ - Adam M. Brenner ${ }^{6}$. \\ Laurence B. McCullough ${ }^{1}$
}

Received: 29 February 2016/Accepted: 14 March 2016/Published online: 28 March 2016

(C) Academic Psychiatry 2016

The concept of medicine as a profession is founded on trust, both that physicians will practice and teach to high intellectual standards and that physicians will seek to promote and protect the interests of patients over and above their own interests. These standards define the ethical concept of fiduciary professionalism expressed in part by the professional virtue of integrity. Integrity is the bedrock virtue, and it is defined by a commitment to practice medicine in accord with the standards of intellectual and moral excellence.

It is heartening to know that the concept of medicine as a profession has a history dating back to the eighteenth century, originating from the work of John Gregory (1724-1773) who heavily influenced Thomas Percival (1740-1804) [1]. Percival in turn was a major influence on the first (1847) Code of Ethics of the American Medical Association [1]. It is also heartening to know that the concept of medicine as a profession is not limited to Western culture but is a transnational and transcultural concept that applies to all levels of medical practice, research, and education today [2]. Maintaining the public's trust in the medical profession requires an ongoing active commitment to the professional

John H. Coverdale

jhc@bcm.edu

Baylor College of Medicine, Houston, TX, USA

2 Stanford University, Stanford, CA, USA

3 Wayne State University, Detroit, MI, USA

4 Harvard Medical School, Boston, MA, USA

5 University of Hawai'i John A. Burns School of Medicine, Honolulu, HI, USA

6 University of Texas Southwestern Medical Center, Dallas, TX, USA virtue of integrity by individual practitioners supported by organizational culture of professionalism. Healthy medical organizations, as public trusts and not merchant guilds, similarly limit self-interest including financial self-interest because they exist primarily for the benefit of patients and their communities [3].

Individual practitioners and organizational and educational leaders are responsible for the hard work of preventing an erosion of professional standards and for regulating the culture of medicine. Healthy organizations demonstrate their commitment to professionalism by learning from reports of wrongdoing how to prevent them from recurring. One goal is to prevent the potential for wrongdoing proactively and nonjudgmentally in order to reduce the need for disclosure and intervention. Should preventive measures fail, the second measure requires authorization for formal reports or "whistleblowing."

Whistleblowing constitutes one among many important methods of self-regulation within the profession, an approach that exists for maintaining and improving professional standards. In general, a whistleblower is a person who reports observed wrongdoing $[4,5]$. The assumption we make is that the person who makes such a report does so honestly, out of respect for the standards of the field and with compassion for the individual or individuals who may be hurt, e.g., a patient or a student, and with a concomitant compassion for and sensitivity to the alleged wrongdoer. The person reporting wrongdoing may have to confront a culture that is permissive of the behavior of concern or that is resistant to adopting the changes necessary to address the concern. While "whistle-blowing" may seem to have negative connotations, we need to fully embrace the concept of self-regulation if we are truly serious about creating mandated, publicly accountable clinical learning environments grounded on patient safety and healthcare quality. 
In this issue of Academic Psychiatry, Hodges et al. [6] presented results of a survey of fourth year medical students' attitudes toward peer accountability from 24 medical schools ( $81 \%$ response rate). They found that $82 \%$ of respondents agreed that they felt professionally obligated to report peers whose personal behaviors compromised their professional responsibilities and that $84 \%$ agreed that they felt professionally obligated to report peers who they believed were seriously unfit to practice medicine. One limitation of these findings, however, is that what should be reported was not discussed or defined.

Our goal for this editorial, therefore, is to discuss some examples of problematic professional behaviors that medical students might confront and the possible role of disclosure and intervention in those circumstances. Illustrative examples of problematic behaviors include a failure to disclose medical errors, cheating in medical school, boundary violations including sexual boundary violations, unprofessional behavior of other student residents and attending faculty, improperly disclosing patient information, posting confidential information on social media, and making derogatory remarks about patients, peers, and supervisors, and withstanding derogatory or demeaning comments directed to medical students by a clinician or educator. By this process, we aim to identify some of the implications for educational leaders of students reporting wrongdoing and to suggest that this is a topic that should be formally addressed in medical schools. Psychiatric educators have an important leadership role to play in helping medical students learn about the importance and practical issues of such reporting and in dealing with their sometimes highly emotional consequences.

\section{Examples of Behaviors That May Compromise Professional Responsibilities}

Medical errors are a common feature of medical practice, and presumably, all well-intentioned physicians will commit errors at some time during their careers. Medical errors are not necessarily a sign of negligence, which is a legal finding. Medical errors have been found to occur for a variety of reasons including physician fatigue, poor communication within the healthcare team or during in patient handoffs between physicians, or through other system failures. An additional important concern is that the physician workforce [7], including the psychiatric workforce [8], is aging, and on the occasions when aging is associated with impaired executive functioning, concerns about clinical performance will also likely surface. In one national survey of trainees including residents and medical students [9], just more than half of the medical student respondents had observed a harmful medical error resulting in transient or permanent injury to patients. Failure to disclose a medical error, at least when a patient suffers significant harm, is not in accord with professional obligations.
Full disclosure of medical errors, at least those that cause significant harm to patients, has been justified on the grounds that disclosure demonstrates respect for patients and that it is the patient's right to know the truth [10-12]. Disclosure represents virtuous behavior $[4,13]$ that promotes patients' trust [14] and their emotional healing [15]. Disclosure to clinical leadership allows for an analysis of the seriousness and root causes of the error and facilitates the prevention of re-occurrences. Some have argued that full disclosure involve not only medical errors that cause harm but even minor errors or "near misses" during the course of medical care. Such cases might involve missing an ordered medication or administering an incorrect medication.

Related ethical guidance is provided by the American Psychiatric Association's principles of medical ethics which enjoins members to be honest in all professional interactions and to strive to report physicians deficient in character or competence and those who engage in fraud or deception to appropriate entities [16]. In addition the joint commission and some state departments of health require hospitals to report serious mishaps [17].

On the other hand, there are a myriad of factors that may inhibit the disclosure of an error by medical students. Examples include a fear of possible retaliation by those in authority, a lack of self-confidence, a wish to fit in with the team, or a sense of discomfort or distress in reporting [18]. Retaliatory factors often include fear of lowered grades, negative comments that will be passed on leaders of other clinical rotations, poor evaluations on Dean's letters, or even worries that disclosures may threaten acceptance into desired residencies. Medical students quickly learn that "affability" is highly prized on the wards and regarded with enthusiastic comments in evaluations. In that regard, medical students may pay a price even when residents and attendings have no explicit intention to retaliate. Other factors include a view that it may not benefit or even may harm the patient or the professional relationship to disclose $[9,10,19,20]$. Students may also be concerned that the patient may not understand the information presented or that the patient would not want to know [19], and many students may not have received training on how to disclose errors [19].

Cheating in medical school is illustrated by a variety of behaviors such as taking credit for another person's work, falsifying data, copying or allowing another student to copy during an examination, or reporting a physical examination finding as normal when not done. There is a paucity of data on cheating behaviors by medical students, especially outside of North America [21], and formal survey definitions of cheating vary [22]. Some surveys, however, indicate that many students had reported engaging in one form of cheating during medical school [23-25], and in one multisite survey of 31 schools, $39 \%$ of students reported having observed an episode of cheating [23]. Unprofessional behaviors in medical 
school limit the validity of assessments of competency, and they are associated with subsequent disciplinary actions against practicing doctors [26].

Although there are many forms of professional boundary crossings and violations [27, 28], one clear unprofessional behavior is engaging in sexual contact with one's own patient. We are not aware of surveys that report on medical student's knowledge of others who have engaged in sexual contact with a current patient, although medical students may themselves hold problematic or worrisome attitudes [29]. A national survey of physicians across several specialties, however, indicated that a substantial minority had personal knowledge of sexual contact by colleagues concurrent with treatment [30] and an important minority of physician respondents has themselves reported engaging in sexual contact with patients [30]. Disciplinary actions by a state medical board provide additional evidence that such involvement does occur [31]. We are also not aware of studies that ascertain the rate of medical student sexual involvement with patients. Sexual relationships with patients are absolutely not permitted by the psychiatry profession's code of ethics $[16$, p4]. Sexual involvement between physicians and patients violates the foundation of trust that is necessary for the therapeutic alliance, disrupting the obligations to patients generated by the ethical principles of respect for autonomy and beneficence. Sexual involvement between physicians and patients does not reflect the virtues of integrity, self-effacement, and self-sacrifice that obligate medical professionals to put aside, and never to act on, feelings of sexual attractiveness in the context of the physician-patient relationship [32].

With respect to other forms of misconduct that may be more common, medical students may be the recipient of a detrimental or derogatory comment by another team member or may witness other team members being demeaned. A persistence of those behaviors is a form of harassment or bullying. A form of pimping called malignant pimping is abusive when intended to reinforce hierarchy while serving the ego of the teacher and when intended to humiliate the student [33]. In one survey, sizeable proportions of medical students, especially those in later clinical years, had been humiliated or degraded, yelled or sworn at, or had experienced discomfort listening to sexual humor [34]. In another survey of six medical schools, nearly all medical student respondents had heard physicians refer derogatorily to patients [35]. Disrespecting learners, colleagues, or patients is wholly antithetical to professional behavior. Calling out colleagues who act disrespectfully is a way of bringing a light to the "dark side of the profession" [36]. Medical students are often reluctant to report abuse, even though the Liaison Committee on Medical Education requires that mechanisms for reporting violations of the standards of conduct of the teacher-learner relationship be investigated without fear of retaliation.

Silence in response to observable erratic and impaired behavior in a medical student peer was identified as a serious concern in an early study of medical student health issues performed by one of us (LWR) with colleagues [37]. In this project involving 955 students from nine medical schools ( $52 \%$ response rate), we posed two hypothetical scenarios: a medical student who is clearly showing erratic behavior and insufficient clinical care practices on a fourth year clinical rotation due to a substance-related health problem and the exact same pattern due to uncontrolled diabetes. For the scenario in which a substance-related health problem was the identified cause, $55 \%$ of student respondents would "tell no one" and for the scenario in which uncontrolled diabetes was identified as the cause, $51 \%$ would "tell no one." This multisite project also revealed that students were sensitive to stigma associated with having even routine health needs. The majority of students in the study feared academic jeopardy - either a harsh evaluation from a clinical supervisor or a weak "Dean's letter," potentially damaging current or future academic standing while in training. These fears were greatest for the most stigmatizing health conditions rather than, for example, the most potentially disabling health conditions, and students consistently preferred care outside of their home institution for stigmatizing conditions. Similar stigmatizing attitudes have also been found among residents [38]. It appears that fears associated with identifying and reporting impairment in colleagues begin early in physician training, and these issues bear careful consideration in fulfilling the responsibility of self-governance by the profession.

\section{Implications of Professional Self-Regulation for Educational Leaders}

The concept of the profession of medicine as a public trust necessitates that the profession regulate itself because in a public trust, professional self-regulation is ethically obligatory. Members therefore should report those who are clinically incompetent as well as those who violate established ethical boundaries by such egregious acts as cheating in medical school, boundary violations including sexual boundary violations, and derogatory or demeaning comments directed to medical students by a clinician or educator. Such reporting demands the virtues of courage and self-sacrifice because whistleblowers are vulnerable to becoming ostracized or punished. Educational leaders should demonstrate clearly articulated means of protecting medical students. In turn, medical students should have faith that medical schools will act responsibly in investigating concerns or complaints.

Medical errors exist on a continuum of seriousness and likely have many determinants including contributing institutional habits and practices. Medical students might be limited in their ability to judge the causes, clinical implications and seriousness of errors committed by more senior professionals. Monolithic standards about when and to whom to disclose 
should therefore not apply. An important first step in learning about the circumstances at play is to initiate a discussion with the physician concerned or with other team members. Such a discussion might establish that further disclosure is not indicated with the patient concerned or with leadership. It might also lead to a decision by another more senior member of the team to do the reporting. These processes can be informed by policy, ethical standards, and guidelines.

Another method of educating students about form, function, and subtleties of disclosure is including students in morbidity and mortality conferences, as well as on committees investigating incident reports. In these forums, leaders should consider not only the standards of care but also how failures were managed and disclosed to injured parties. By gently referring to examples from other disciplines (such as the airline industry) where transparency and disclosures of errors and near misses are routine and essential components of creating and maintaining culture of quality and safety, leaders can encourage active participation by removing the fear and stigma that often accompany such discussions.

Medical students and their teachers should also appreciate that an association has been found between the committal of errors and burnout [25] and depression [39]. These considerations, along with a recognition that all medical professionals will at least at one time err, should facilitate a compassionate response to the committal of errors. Educational leaders should role model such a compassionate response and cultivate openness to discussing errors and the factors that may contribute to them. Such demonstrations of compassion and openness are not easy work because acceptance of one's human failings is difficult and cultivating such transparency can be emotionally painful for all concerned. A preferred response to an error is to assume positive intentions, show respect, and engage in shared accountability, while seeking not to evoke shame and guilt [40]. The goal should be contribute to professional formation by helping students and colleagues to become their best selves. One pervasive problem in many medical settings is the lack of regularly held forums to address these issues. Far too often, the pace of patient care thwarts open and candid discussion and there are sometimes only perfunctory sessions in medical school and residency on the wide variety of circumstances that lead to impaired performance.

Thus, educational leaders should establish opportunities to formally teach how to approach medical errors and to integrate this teaching into a broader curriculum of patient safety and error prevention [19, 41]. Students should be helped to learn from and cope with medical errors [42] and should see in real time how teams and leaders take accountability for medical errors and full disclosure. Teaching has been shown to improve learners' knowledge, skills, and attitudes to error disclosure [43]. Role-modeling, including a willingness to accept responsibility for an error and to disclose and apologize for it, can influence students' attitudes and behaviors [9]. Institutions should also look to address the emotional needs of the professionals who are involved in medical errors [44, 45]. The formal and especially the informal medical curriculum should be routinely evaluated for how it supports or hinders ethically guided professional decision-making in these processes [2].

Maintaining and strengthening professional integrity is crucial to the provision of an excellent standard of quality of care and medical education and to promoting a culture of respect and responsibility to every student, colleague, and patient. All of us, at all professional stages and at all levels of seniorityincluding medical students - should be vigilant to recognize the factors and deficiencies that may erode the quality of clinical care and should speak up about them. We are therefore encouraged by the finding reported here [6] that the majority of medical student respondents appeared to be willing to report peers who were compromised in their professional abilities. Doing so is a matter of professional responsibility that serves to maintain and promote the foundation of public trust in the medical profession.

\section{Compliance with ethical standards}

Disclosure On behalf of all authors, the corresponding author states that there is no conflict of interest.

\section{References}

1. McCullough LB. John Gregory (1724-1773) and the invention of professional medical ethics and the profession of medicine. Dordrecht: Kluwer; 1998.

2. Coverdale J, McCullough LB. Professionalism in medicine as a transnational and transcultural ethical concept. In: Roberts LW, Reicherter D, editors. Professionalism and ethics in medicine. New York: Springer Science and Business Media, LCC; 2015. Provide pages.

3. Chervenak F, McCullough LB. The diagnosis and management of progressive dysfunction of health care organizations. Obstet Gynecol. 2005;105:882-7.

4. Bolsin S, Faunce T, Oakley J. Practical virtue ethics: healthcare whistleblowing and portable digital technology. J Med Ethics. 2005;31:612-8.

5. Bolsin S, Pal R, Wilmshurst P, Pena M. Whistleblowing and patient safety: the patient's or the profession's interests at stake. J R Soc Med. 2011;104:278-82.

6. Hodges CE, Tak HJ, Curlin FA, Yoon J. Whistleblowing in medical school: a national survey on peer accountability and professional misconduct in medical students. Acad Psychiatry 2016. 10.1007/ s40596-015-0405-y.

7. Kupfer JM. The graying of US physicians. Implications for quality and the future supply of physicians. JAMA. 2016;315:341-2.

8. Scully JH, Wilk JE. Selected characteristics and data of psychiatrists in the United States, 2001-2002. Acad Psychiatry. 2003; $247-$ 251. 
9. Martinez W, Hickson GB, Miller BM, et al. Role-modeling and medical error disclosure: a national survey of trainees. Acad Med. 2014;89:482-9.

10. Finkelstein D, Wu AW, Holtzman NA, Smith MK. When a physician harms a patient by a medical error: ethical, legal, and riskmanagement considerations. J Clin Ethics. 1997;8:330-5.

11. Sweet MP, Bernat JL. A study of the ethical duty of physicians to disclose errors. J Clin Ethics. 1997;8:341-8.

12. Varjavand N, Bachegowda LS, Gracely E, Novack DH. Changes in intern attitudes toward medical error and disclosure. Med Educ. 2012;46:668-77.

13. Rodulson V, Marshall R, Bleakley A. Whistleblowing in medicine and in Homer's iliad. J Med Humanit. 2015. doi:10.1136/medium2015-010673.

14. Wu AW, Cavanaugh TA, McPhee SJ, Lo B, Micco GP. To tell the truth: ethical and practical issues in disclosing medical mistakes to patients. J Gen Intern Med. 1997;12:770-5.

15. Sanford DE, Fleming DA. We meant no harm, yet we made a mistake; why not apologize for it? a student's view. HEC Forum. 2010;22:159-69.

16. American Psychiatric Association. The principles of medical ethics with annotations applicable to psychiatry. Washington DC: American Psychiatric Association; 2013.

17. Leape LL. Reporting of adverse events. New Engl J Med. 2002;347:1633-8.

18. Martinez W, Lehmann LS. The "hidden curriculum" and residents' attitudes about medical error disclosure: comparison of surgical and nonsurgical residents. J Am Coll Surg. 2013;217:1145-50.

19. White AA, Gallagher TH, Krauss MJ, et al. The attitudes and experiences of trainees regarding disclosing medical errors to patients. Acad Med. 2008;83:250-6.

20. White AA, Bell SK, Krauss MJ. How trainees would disclose medical errors: educational implications for training programs. Med Educ. 2011;45:372-80.

21. Tonkin AL. "Lifting the carpet" on cheating in medical school exams. BMJ. 2015;351:h4014.

22. Kusnoor AV, Falik R. Cheating in medical school: the unacknowledged ailment. South Med J. 2013;106:479-83.

23. Baldwin Jr DC, Daugherty SR, Rowley BD, Schwarz MD. Cheating in medical school; a survey of second-year students at 31 schools. Acad Med. 1996;71:267-73.

24. Coverdale J, Henning MA. An analysis of cheating behaviours during training by medical students. Med Teach. 2000;22:582-4.

25. Dyrbye LN, Massie FS, Eacker A, Harper W, et al. Relationship between burnout and professional misconduct and attitudes among US medical students. JAMA. 2010;304:1173-80.

26. Papadakis MA, Teherani A, Banach MA, et al. Disciplinary action by medical boards and prior behavior in medical school. $\mathrm{N}$ Engl $\mathrm{J}$ Med. 2005;353:2673-82.

27. Gabbard GO, Kassaw KA, Perez-Garcia G. Professional boundaries in the era of the internet. Acad Psychiatry. 2011;35:168-74.
28. Gabbard GO, Nadelson C. Professional boundaries in the physician-patient relationship. JAMA. 1995;273:1445-9.

29. Coverdale J, Turbott SH. Teaching medical students about the appropriateness of social and sexual contact between doctors and their patients: evaluation of a programme. Med Educ. 1997;31:335-40.

30. Bayer T, Coverdale J, Chiang E. A national survey of physicians' behaviors regarding sexual contact with patients. South Med J. 1996;89:997-82.

31. Morrison J, Wickersham P. Physicians disciplined by medical boards and prior behavior in medical school. JAMA. 1998;279: 1889-93.

32. McCullough LB, Chervenak FA, Coverdale J. Ethically justified guidelines for defining sexual boundaries between obstetriciangynecologists and their patients. Am J Obstet Gynecol. 1996;175: 496-500.

33. Reifler DR. The pedagogy of pimping: educational rigor or mistreatment? JAMA. 2015;314:2355-6.

34. Wilkinson TJ, Gill DJ, Fitzjohn J, Palmer CL, Mulder R. The impact on students of adverse experiences during medical school. Med Teach. 2006;28:129-35.

35. Feudtner C, Christakis DA, Christakis NA. Do clinical clerks suffer ethical erosion? students' perceptions of their ethical environment and personal development. Acad Med. 1994;69:670-9.

36. Laine $\mathrm{C}$, Taichman $\mathrm{DB}, \mathrm{LaCombe} \mathrm{MA}$. On being a doctor: shining a light on the dark side. Ann Intern Med. 2015;163:320.

37. Roberts LW, Warner TD, Rogers M, Horwitz R, et al. Medical student illness and impairment: a vignette-based survey study involving 955 students at 9 medical schools. Compr Psychiatry. 2005;46:229-37.

38. Moutier C, Cornette M, Lehrmann J, Geppert C, et al. When residents need health care: stigma of the patient role. Acad Psychiatry. 2009;33:431-41.

39. Fahrenkopf AM, Sectish TC, Barger LK, et al. Rates of medication errors among depressed and burnt out residents: prospective cohort study. BMJ. 2008;336:488-91.

40. Pronovost PJ, Bienvenu OJ. From shame to guilt to love. JAMA. 2015;314:2507-8.

41. Walton M, Woodward H, Staalduinen SV. Republished paper: the WHO patient safety curriculum guide for medical schools. Postgrad Med J. 2011;87:317-21.

42. Martinez W, Lo B. Medical students' experiences with medical errors: an analysis of medical student essays. Med Educ. 2008;42: $733-41$

43. Stroud L, Wong BM, Hollenberg E, Levinson W. Teaching medical error disclosure to physicians-in-training: a scoping review. Acad Med. 2013;88:884-92.

44. Wu AW. Medical error: the second victim. BMJ. 2000;320:726-7.

45. Gallagher TH, Waterman AD, Ebers AG, et al. Patients' and physicians' attitudes regarding the disclosure of medical errors. JAMA. 2003;289:1001-7. 